\title{
Underdetermination of Physical Theory
}

\author{
by \\ Lars Bergström \\ (Stockholm University)
}

\section{Introduction.}

Our theories of the world are related in various ways to experience. We construct theories partly in order to account for what we have observed and partly in order to systematize and support our expectations for future experience. But what we experience is not sufficient to determine our theories. Different theories may account for our observations equally well. This, roughly speaking, is the thesis that physical theory is underdetermined. W.V. Quine has formulated the idea in different ways in different contexts. However, before we consider Quine's formulations, let us look at the following passage from an address delivered by Albert Einstein on the occasion of Max Planck's sixtieth birthday in 1918:

The supreme task of the physicist is to arrive at those universal elementary laws from which the cosmos can be built up by pure deduction. There is no logical path to these laws; only intuition, resting on sympathetic understanding of experience, can reach them. In this methodological uncertainty, one might suppose that there were any number of possible systems of theoretical physics all equally well justified; and this opinion is no doubt correct, theoretically. But the development of physics has shown that at any given moment, out of all conceivable constructions, a single one has always proved itself decidedly superior to all the rest. Nobody who has gone deeply into the matter will deny that in practice the world of phenomena uniquely determines the theoretical system, in spite of the fact that there is no logical bridge between phenomena and their theoretical principles $[\ldots] .{ }^{1}$

In the first half of this passage, Einstein seems to say that physical theory is underdetermined by experience or observational evidence; in the second half, he seems to say that physical theory is nevertheless uniquely determined by "the world of phenomena". Presumably, the term 'phenomena' stands for what we directly observe or experience. Hence, there appears to be a tension between the first and the second parts of Einstein's pronouncement.

It is hard to see how it can be shown in practice that a given theoretical system is superior to every conceivable rival, even relative to a given moment. This may be doubted even if there is always one system which is clearly better than its actual rivals. Neither can

\footnotetext{
${ }^{1}$ Albert Einstein, Ideas and Opinions (New York, Crown Publishers, 1954), p. 226. Incidentally, we may note that Einstein seems to recommend something like the so-called method of Verstehen for use in the Naturwissenschaften.
} 
we assume that the tension in Einstein's pronouncement disappears if we distinguish between actual and possible experience. At any given moment, our data consists in (a subset of) the actual observations of mankind up to this moment. Einstein's point is not that these actual data are insufficient - while all possible data might be sufficient - to determine the theoretical system. On the contrary, he seems to be saying that actual data are sufficient, at least in practice.

Quine suggests, on the other hand, that not even all possible data are sufficient. His claim is 'that scientific theory is under-determined by all possible data; in other words, that different theories can be empirically equivalent' (RA 294). A somewhat earlier formulation is this:

[...] physical theory is underdetermined even by all possible observations. [...] Physical theories can be at odds with each other and yet compatible with all possible data even in the broadest sense. In a word, they can be logically incompatible and empirically equivalent (RIT 178-9).

One puzzling feature of these formulations is that they seem to equate empirical equivalence and compatibility with all possible data. Surely, empirically equivalent theories may be incompatible with certain data. They need not be empirically viable. In a later context, Quine has said that 'it is a poor idea to assume compatibility with all possible data. [...] What matters is that the theories be empirically equivalent' (CB 53).

One might suppose that different theories are empirically equivalent if they have the same empirical content. If so, one version of the underdetermination thesis would be that theories are underdetermined by their empirical content. One and the same empirical content can be embedded within different theoretical superstructures. In Quine's words, 'there are alternative hypothetical substructures that would surface in the same observable ways' (EES 313).

The formulations presented so far may give a sense of what the underdetermination thesis is about. But more needs to be said. For example, more needs to be said about the interpretation of key terms such as 'theory', 'empirical content', and 'empirical equivalence'.

\section{Theories.}

When Quine speaks of 'theories', what he has in mind are usually theory formulations (e.g. TT 24 and PT 96). A theory formulation 'is simply a sentence-typically a conjunctive sentence comprising the so-called axioms of the theory' (EES 318). Obviously, different theory formulations may be logically equivalent, and it should come as no surprise that logically equivalent theories are also empirically equivalent. Similarly with different theory formulations where one is merely a translation of the other. The underdetermination thesis goes beyond this. The relevant kind of difference between the theory formulations in 
question is that we cannot convert one into a formulation logically equivalent with the other by reinterpretation sentence by sentence (EES 320, PT 97). Let us say that theories which are different in this sense are (mutually) irreconcilable. Theories which are irreconcilable cannot be regarded as mere notational variants of one and the same theory.

Quine sometimes says that physical theory is underdetermined, but in other places he says that physical theories - in the plural - can be underdetermined. He is sometimes taken to hold that all theories, or all physical theories, are underdetermined, but this interpretation should be rejected. Thus, in a reply to a paper by W.H. Newton-Smith, Quine writes the following:

\footnotetext{
He [Newton-Smith] begins with a reference to 'Quine's notorious claim that [...] all theories are underdetermined, [...]'. [...] I conjectured that physical theory, the global system of the world, is underdetermined, but not that every subordinate system was underdetermined (CNS 66).
}

This indicates that Quine takes the underdetermination thesis to apply primarily to our global system of the world. One gets the same impression from several other passages. Sometimes he suggests that a "global theory" or "system of the world" is a theory which can account for all observable events (see e.g. EES 313 and 327). However, if the thesis is restricted in this way, it is perhaps less interesting, since we might never come across a theory which is global in this sense. Rather I think we should take the underdetermination thesis to apply to theories which are global in the sense that they formulate the totality of someone's (explicit or implicit) beliefs at some time about the world. Such theories also include large parts of mathematics, 'for mathematics infiltrates all branches of our system of the world' (PT 15).

At one point, Quine asks us to imagine 'an exhaustive encyclopedic formulation of our total scientific theory of the world' (TT 28). Such a formulation would be a global theory in the relevant sense. This is what Quine refers to by the phrase 'physical theory'; he is not merely concerned with theories of phenomena which are professionally studied by physicists. The underdetermination theory says that global theories are underdetermined. However, there is no need to exclude theories which can account for all observable events but which are never formulated or believed. Let us call these too 'global'. We may recall that Einstein was concerned with possible 'systems of theoretical physics', systems which contain those 'universal elementary laws from which the cosmos can be built up by pure deduction'. If the cosmos contains everything that exists or happens in the world, such systems should also be regarded as global in what I take to be Quine's sense. But maybe Einstein was thinking of physical theories in a more narrow sense.

However, I suggest that the underdetermination thesis should also be taken to concern less global theories. I think this is in accordance with Quine's intentions. He sometimes gives examples of underdetermined theories - such as 'Riemannian and Euclidean 
geometry as applied to the surface of a sphere' (PT 96) - which are certainly not global. I believe his claim is that such "subordinate" theories can also be underdetermined. As indicated by several of the quotations given above, I believe his underdetermination thesis says two things: that our global theory of the world is underdetermined and that other, more subordinate theories can be underdetermined.

\section{Empirical content.}

Intuitively, the empirical content of a theory is what the theory says or implies about observable features of the world. Observable features of the world are described by means of observation sentences. According to Quine, an observation sentence for a given speech community is an occasion sentence which is directly and firmly associated with sensory stimulations for every member of the community and on which all members give the same verdict when witnessing the same situation (PT 3). (Clearly, this is an idealization. People are sometimes mistaken about the truth-value of observation sentences. Possibly, no sentence satisfies Quine's definition, strictly speaking. ${ }^{2}$ ) Examples of observation sentences are 'It's cold', 'That is a dog', 'This is a flower'. Most observation sentences report physical things and events, but some, e.g. 'Tom perceives a dog', are mentalistic (PT 62).

Observation sentences are occasion sentences, i.e. they are true on some occasions and false on others. Therefore, they cannot be implied by scientific theories, which are either true or false once and for all. However, two observation sentences can be combined into a general sentence of the form 'Whenever this, that'. An example would be 'Whenever there is a raven, it is black', or simply 'All ravens are black'. Such sentences are called observation categoricals; they are true or false once and for all, and they can be implied by scientific theories. An observation categorical is synthetic for a given speaker if the stimulations associated with the antecedent are not completely included among the stimulations associated with the consequent. Synthetic observation categoricals can be tested in experiments. Two observation categoricals are synonymous for a speaker if their respective components are associated with the same stimulations. The empirical content of a theory for a given speaker consists of the set of synthetic observation categoricals implied by it, plus all synonymous ones. Moreover, Quine says that two theories are empirically equivalent for a given community if they have the same empirical content for each member (PT 16-7). Presumably, the empirical content which is common to the two theories can be different for different speakers. Therefore, it is unclear what would be the empirical content of a theory for a given community. Maybe we could say that it is the union-or the intersection? - of the empirical contents for all members.

\footnotetext{
${ }^{2}$ In one place, Quine says that 'even a common observation term such as 'blue' has its penumbra of vagueness, where witnesses may disagree in their verdicts. The really distinctive trait of observation terms and sentences is to be sought not in concurrence of witnesses but in ways of learning. Observational expressions are expressions that can be learned ostensively' (EES 316).
} 
Notice that, on this account, the empirical content of a theory is relative to speakers or communities. It seems that it is also relative to times, since the stimulations associated with an observation sentence for a speaker can be expected to vary from time to time.

Quine claims to have defined empirical content only for testable theories, i.e. for theories which imply some synthetic observation categoricals (PT 16, 95). But he would probably allow for the possibility that some untestable theories have empirical content. He simply says that he has no definition of empirical content to offer for such theories (PT 95). Maybe the idea is that an untestable theory can somehow contribute to the empirical content of some more comprehensive theory of which it is a part, and that this gives it an empirical content even though it does not by itself imply any observation categoricals. ${ }^{3}$ But if theories which do not imply any synthetic observation categoricals can have empirical content, it seems rather strange to say that the empirical content of a testable theory is exhausted by the set of synthetic observation categoricals implied by it (plus all synonymous ones). If untestable theories can get empirical content in some other way, it seems reasonable to assume that the same is true for testable theories. But then the empirical content of a testable theory should not be defined in the way Quine suggests.

\section{Empirical equivalence.}

Quine sometimes seems to identify empirical equivalence and sameness of empirical content. However, he notes that 'much solid experimental science fails of testability in the defined sense' (PT 95). Therefore, he also has a more general definition of empirical equivalence, according to which two theories are empirically equivalent when 'whatever observation would be counted for or against the one theory counts equally for or against the other' (PT 96). As far as I can see, there is no guarantee that testable theories which have the same empirical content are also empirically equivalent in this general sense.

Let me try to illustrate this by means of an example. Let $R$ be the general theory of relativity. I take it that $R$ is not by itself testable in Quine's sense. A lot of other theories and auxiliary hypotheses, including mathematical ones, are needed to derive synthetic observation categoricals. If $A$ is the conjunction of these auxiliary theories, the compound theory $R A$ is testable. ( $R A$ is the conjunction of $R$ and $A$.) Now let $C$ be Gödel's axiom of constructibility, ' $V=L$ ', which says that all sets are constructible. I assume that $C$ is not implied by $R A$; it is not needed to derive observation categoricals from general relativity and it is not part of ordinary set theory. If $C$ is added to $R A$ we get $R A C$. Presumably, $R A$ and $R A C$ have the same empirical content (for the scientific community at a given time). $C$ does not add anything to the empirical content of $R A$. But we would probably not say that

\footnotetext{
${ }^{3}$ However, as Quine points out, we cannot assume that an untestable sentence has empirical content if it implies synthetic observation categoricals in conjunction with some other untestable sentence, for every sentence satisfies this requirement. For example, let $Q$ be Russell's nonsense sentence 'Quadruplicity drinks procrastination' and let $O$ be any synthetic observation categorical. Now, neither $Q$ nor $(Q \supset O)$ is testable, but their conjunction is testable (see SS 48).
} 
whatever observation would be counted for or against $R A$ would count equally for or against $R A C$. Presumably, $R A$ and $R A C$ are empirically equivalent in one of Quine's senses, but not in the other.

Let me explain. I assume that $R A$ and $R A C$ are empirically equivalent in the sense that they imply the same synthetic observation categoricals. Then there are three different possibilities. (1) Either $R A$ is a better theory than $R A C$ by our scientific standards. Maybe it is better because it is "simpler". If so, we may say that $R A$ is a better explanation of available data and that, as far as we can see, whatever observation would be counted for $R A$ counts much less or not at all for $R A C$. (2) Or else $R A C$ is a better theory than $R A$, perhaps because there are mathematical or philosophical reasons for adding Gödel's axiom to the mathematical parts of $A .^{4}$ If so, we might say that $R A C$ is a better explanation and that whatever observation counts for $R A$ counts more for $R A C$. In cases (1) and (2), $R A$ and $R A C$ are not empirically equivalent in Quine's more general sense. But there is also a third possibility. (3) $R A$ and $R A C$ are equally good theories according to our scientific standards. If so, we have a case of underdetermination. We have two irreconcilable theories which are empirically equivalent-not merely in the sense that they imply the same synthetic observation categoricals, but in the general sense that 'whatever observation would be counted for or against the one theory counts equally for or against the other'. In Einstein's words, the two theories are 'equally well justified'.

I do not think that (3) is a very realistic alternative. I believe that (1) is the actual case. It also seems to me that (1) is not a very interesting case of underdetermination. After all, in case (1), $R A$ is a better explanation than $R A C$ of the available data. Consequently, sameness of empirical content is not the most interesting kind of empirical equivalence. Quine's general sense of 'empirical equivalence' is more relevant in the present context. Another illustration of this point is where two theories imply the same synthetic observation categoricals, but where one of them fits much better than the other into a larger system of theories for which there is independent empirical support. That such cases may occur is one reason why Quine often limits his discussion to global theories, 'so that there is no question of fitting the rival theories into a broader context' (PT 98). He seems to agree that such cases do not exemplify underdetermination in his sense.

Now, since the relevant sense of 'empirical equivalence' makes empirical equivalence depend upon the specific scientific virtues which make some theories better than others, something needs to be said about these and about the notion of scientific value.

\footnotetext{
${ }^{4}$ Quine says that considerations such as 'simplicity, economy, and naturalness [...] contribute to the molding of scientific theories generally', and that they support Gödel's axiom of constructibility (PT 95). So maybe our global system of the world should contain Gödel's axiom. Notice, that our system of the world may contain a lot of sentences which have very little to do with empirical content. Quine writes: 'Much that is accepted as true or plausible even in the hard sciences, I expect, is accepted without thought of its joining forces with other plausible hypotheses to form a testable set. Such acceptations may be prompted by symmetries and analogies, or as welcome unifying links in the structure of the theory. [...] Having reasonable grounds is one thing, and implying an observation categorical is another' (SS 49).
} 


\section{Scientific value.}

Quine says that his general sense of 'empirical equivalence' is 'ill-defined' (PT 96). The reason, presumably, is that we have no precise standards of empirical justification or scientific value. In one place, Quine says that scientific method 'is a matter of being guided by sensory stimuli, a taste for simplicity in some sense, and a taste for old things' (WO 23). This is rather vague. In another context he lists the following virtues that scientific hypotheses can have in varying degrees: conservatism, modesty, simplicity, generality, refutability, and precision (WB 66-79 and 98). Other philosophers have mentioned the same and/or similar scientific virtues. For example, Thomas Kuhn says that

five characteristics-accuracy, consistency, scope, simplicity, and fruitfulness - are all standard criteria for evaluating the adequacy of a theory. [...] Together with others of much the same sort, they provide the shared basis for theory choice. ${ }^{5}$

Quine seems to regard his virtues as means to predictive efficacy (see WB 135), but he also says that prediction is not the main purpose of science. 'One major purpose is understanding. Another is control and modification of the environment' (PT 2). Kuhn, on the other hand, regards his five characteristics more as constitutive of the goal or purpose of science. $^{6}$

It would be generally admitted, I believe, that there is no algorithm for determining the scientific value of a theory-i.e. the degree to which the theory has the good-making characteristics of a good theory. ${ }^{7}$ But most philosophers of science would agree that the scientific value of a theory depends upon features of the kind mentioned by Quine and Kuhn. The terms used to indicate such features are vague and ambiguous. Moreover, competent scientists may disagree about the relative importance of the various features and about the way they should be aggregated in particular cases. In practice, different opinions within the scientific community may usually tend to converge, in due course, towards a common verdict. But this does not show that the common verdict is correct in an objective sense. Presumably, in many cases there is no fact of the matter independently of the very verdict arrived at by scientists. Rather, a consensus within the scientific community that one theory is better, or simpler, than another can be explained (within limits) by reference to ordinary group-psychological mechanisms. As long as a group of people believe that there

\footnotetext{
${ }^{5}$ T.S. Kuhn, 'Objectivity, Value Judgment, and Theory Choice', in: The Essential Tension (Chicago, University of Chicago Press, 1977), 320-39, p. 322.

${ }^{6}$ For arguments and further clarification concerning this claim and other points made in this section, see my paper 'Scientific value', International Studies in the Philosophy of Science, vol. 10, no. 3 (October 1996), 189202.

${ }^{7}$ For example, Quine writes: 'No general calibration of either conservatism or simplicity is known, much less any comparative scale of the one against the other' (SS 49).
} 
is a correct answer to a question, they tend to work their way to a consensus, even if there is no sufficient evidence one way or the other, and even if many different answers are in fact equally possible. Consider e.g. the classic experiments with the so-called autokinetic effect. When a spot of light is projected in a totally darkened room it will appear to move, but different individuals will perceive very different movements. However, when they are asked to describe the movement of the spot to the other members of a group, their judgments soon tend to converge towards a group norm. It seems that a consensus concerning the overall scientific value of a theory may arise in a similar way.

With reference to considerations of this kind, I suggest that there is often no fact of the matter as to whether two theories are "equally good" or "equally justified". Consequently, there is often no fact of the matter as to whether two theories are empirically equivalent in Quine's general sense either. This in turn leaves room for another psychological mechanism. ${ }^{8}$ Scientists can be expected to dislike situations in which two irreconcilable theories are equally good. Such a situation would make scientific life more difficult. Therefore, whenever a situation of this kind appears to obtain-and especially if the two theories also appear to be logically incompatible - scientists can be expected to make it their business to show that one of them is better after all. And they will not give up until they succeed (by their own lights). Indeed, this may explain Einstein's observation that 'out of all conceivable constructions, a single one has always proved itself decidedly superior to all the rest.'

But if this is right, it undermines Quine's underdetermination thesis. The relevant kind of empirical equivalence can hardly be expected to obtain. Quine says that

\footnotetext{
we have no reason to suppose that man's surface irritations even unto eternity admit of any one systematization that is scientifically better or simpler than all possible others. It seems likelier, if only on account of symmetries and dualities, that countless alternative theories would be tied for first place (WO 23).
}

As far as I can see, this is not likelier. Rather, it seems very unlikely. Empirical and methodological considerations can never force the scientific community to conclude that two theories are tied for first place, and psychological mechanisms can be expected to work against such a conclusion. Moreover, there is no fact of the matter about which the scientific community would then be mistaken.

\section{Weak underdetermination.}

However, at this point we may return to Quine's other notion of empirical equivalence, i.e. sameness of empirical content. In order to avoid the problem that 'empirical content' is only

\footnotetext{
${ }^{8}$ See also my paper 'Quine, underdetermination, and skepticism', The Journal of Philosophy, vol. 90, no. 7 (July 1993), 331-358, p. 339.
} 
defined for testable theories, let us restrict ourselves to such theories. In particular, let us focus on global theories. Let us also presuppose a certain community and time in order to bypass the relativity of empirical content.

The underdetermination thesis might now be taken to say that irreconcilable global systems of the world may have the same empirical content. However, this is not a very interesting claim. Thus, for example, if $G$ is our global system of the world (at a given time) and $C$ is Gödel's axiom of constructibility, then presumably $G$ and $G C$ have the same empirical content and are irreconcilable-I assume that $C$ is not implied by $G$-but they are not different in a very interesting way. They have too much in common, theoretically.

One might require that the theories in question are tight in the sense that they are not the result of adding to another theory some theoretical sentence which does not affect the empirical content or the explanatory power of the original theory. ${ }^{9}$ Thus, for example, it might be argued that $G$ and $G C$ are not serious rivals in the sense of the underdetermination thesis, since $G C$ is not a tight theory.

However, I suggest that a better alternative is to say that underdetermination is a matter of degree. Even in the case of $G$ and $G C$ there is a certain small amount of underdetermination, but this is almost negligible. More interesting cases of underdetermination are those in which theories with the same empirical content are not only irreconcilable-in the sense that we cannot convert one into the other by reinterpretation sentence by sentence, which is the sense Quine has in mind-but also have theoretical contents which are "very different". This is certainly vague, but that should not be a big problem if underdetermination is a matter of degree. In general, we may say that the degree to which a given theory is underdetermined is the degree to which it is theoretically different from irreconcilable theories with the same empirical content. ${ }^{10}$ It may not be an easy task to define a plausible and precise scale on which the theoretical difference between theories can be measured, but I think the intuitive idea is clear enough.

Now, if our global system of the world has the same empirical content as some (possibly unknown) system which is theoretically very different from ours, this fact would certainly be interesting. It would be interesting even if one of the systems-ours, for example-

\footnotetext{
${ }^{9}$ The notion of tightness is employed in my paper 'Underdetermination and realism', Erkenntnis, vol. 21 (1984), 349-65, p. 351. It is based upon a rather similar idea of Quine's (EES 323) that the addition to a theory of 'some gratuitous further sentences that had no effect on its empirical content' is not sufficient for underdetermination. Notice, that if theories $A$ and $B$ are irreconcilable and have the same empirical content, each has the same empirical content as the disjunctive theory $A \vee B$, which is logically weaker. Are $A$ and $B$ 'gratuitous further sentences', in Quine's sense, in relation to $A \vee B$ ? Suppose further that the empirical content is a finite set of synthetic observation categoricals. Does it follow that $A, B$, and $A \vee B$ are all 'gratuitous further sentences' in relation to the observation categoricals? In one place, Quine says that 'gratuitous branching of theories' is of 'no interest to the thesis of under-determination' (EES 323), but it is not so clear what a 'gratuitous branching' is. We may suppose that a branching is 'gratuitous' if it is scientifically worthless, but that does not help much.

${ }^{10}$ It may be tempting to add that $G C$ is more underdetermined than is $G$, since it is logically stronger but has the same empirical content. However, I shall disregard this idea.
} 
seems to us to be scientifically better or simpler than the other. Let us call this weak underdetermination. This is probably not the kind of underdetermination Quine has in mind most of the time, but it is perhaps just as interesting. If I am right, it may even be more interesting because it is more realistic. Also, the higher degree to which a given theory is underdetermined, the more interesting is the fact that it is underdetermined. Or so it seems.

\section{Logical incompatibility.}

Is it possible that irreconcilable theories with the same empirical content are logically incompatible? We have seen that Quine holds that physical theories 'can be logically incompatible and empirically equivalent' (RIT 179). On the other hand, Michael Dummett has argued that this claim 'is absurd, because there could be nothing to prevent our attributing the apparent incompatibility to equivocation'. ${ }^{11}$ In 1975, Quine 'took up the question of equivocation' (see CNS 67), but even then he seems to believe that some incompatibility may remain when the idea of equivocation has been fully exploited (EES 326-7). However, in later works, under the influence of Donald Davidson, he seems to have come closer to Dummett's position. The argument runs as follows. If two incompatible theory formulations have the same empirical content, the incompatibility can only concern purely theoretical sentences. For example, suppose that one theory says that neutrinos have mass and the other theory says that neutrinos do not have mass. ${ }^{12}$ This looks like a contradiction, but the incompatibility can easily be avoided if we assume that the word 'neutrino' does not have the same meaning in the two theories; to mark the difference we may even change the spelling to 'neuttrino' in one of the theories (TT 29, PT 97-8). Of course, we may also assume that the word 'mass' has different meanings in the two theories. Other apparent incompatibilities are treated in the same way.

So far, so good. But can we also rule out the possibility that the words 'neutrino' and 'mass' have the same meanings in the two theories, so that there is a real incompatibility? Is it 'absurd' to assume that the two theories are logically incompatible, as Dummett says? It seems that Quine would not go this far. In 1990 he gives an example of empirically equivalent theories which are incompatible:

An example is Poincaré's, in which he contrasts our common-sense infinite space and familiar rigid bodies with a finite space in which those bodies shrink as they move away from center. The two theories are clearly empirically equivalent but logically incompatible (CB 53). ${ }^{13}$

\footnotetext{
${ }^{11}$ Michael Dummett, Frege: Philosophy of Language (London: Duckworth, 1973), p. $617 \mathrm{fn}$.

${ }^{12}$ The example of neutrinos and mass is discussed by Quine in WO 16 and SS 70.

${ }^{13}$ On the other hand, this particular example may not be quite convincing. At roughly the same time, or slightly later, Quine says that the two theories in Poincaré's example are irreconcilable but logically compatible (PT 97).
} 
He goes on to say that 'in the case of incompatibility we can shift to a theory formulation that operates in the same way and is compatible' (CB 53). Still, it seems that, on Quine's view, empirically equivalent theories can be logically incompatible. Another example of this might be $G C$ and $G \neg C$, i.e. our global system of the world in conjunction with the constructibility axiom and its negation, respectively. Surely, it is very natural to think of $G C$ and $G \neg C$ as incompatible theories.

However, from a Quinean point of view, there appears to be another argument which might be used to show that $G C$ and $G \neg C$ are not incompatible. Of course, they are syntactically incompatible, but they are not incompatible in the sense that both cannot be true. The reason is that the meaning of theoretical sentences is immanent or relative to a theory. Quine writes:

Unless pretty firmly and directly conditioned to sensory stimulation, a sentence $S$ is meaningless except relative to its own theory; meaningless intertheoretically (WO 24).

Therefore, one might conclude, the sentence $C$ does not have the same meaning in $G C$ and in $G \neg C$, and similarly, for that matter, with $G$. Consequently, the two theories are not logically incompatible (in the sense that both cannot be true). In Dummett's words: the claim that they are empirically equivalent and logically incompatible 'is absurd'.

This argument is too legalistic. In some sense, and to some extent, a sentence may be meaningless except relative to its own theory, but this need not make it unintelligible from the point of view of other theories. Suppose I accept $G C$ and you accept $G \neg C$. If we want to understand one another, each of us must device a translation manual from the other's idiolect into his own in order to understand the other's theory. Surely, a very natural manual for me is to translate $G \neg C$ in your idiolect into $G \neg C$ in my idiolect. In other words, I assume that we use language in the same way. If this translation manual works-i.e. if it leads to 'fluency and effectiveness of dialogue' (PT 59) - then it is OK. Nothing more can be asked for, and relative to this translation manual the two theories are indeed logically incompatible. In fact, even if the manual does not work, by applying it I have formulated a theory in my own language which is incompatible with my theory. Surely, there is nothing 'absurd' about this.

In principle, however, there may be other translation manuals from your idiolect to mine. For some such manual, $\neg C$ in your idiolect is compatible with $C$ in mine. For example, on such a manual, the word 'set' in your idiolect does not mean the same as 'set' in mine. Consequently, $G$ does not mean the same for you and me either. Presumably, the word 'set' is connected to a lot of mathematical and set-theoretical sentences in $G$. But if these sentences do not mean the same for you and me, and if my translation of $\neg C$ in your idiolect is compatible with $C$ in mine, presumably our dialogue is not fluent and effective. The reason is that my translations of simple sentences in your idiolect would quite often be 
very complicated. I might simplify such translations by defining new terms in my idiolect, but this would complicate my vocabulary. Alternatively, I might coin new undefined words in my idiolect as translations of some of your words-e.g. I may translate your word 'set' as 'sett', which is a new word in my idiolect-but it seems to me that this is really no translation, since I do not use the word 'sett' at all, except as a translation of your 'set'. So maybe this kind of translation manual is not acceptable. If so, Quine's way of getting rid of incompatibilities is perhaps not so unproblematic after all.

A more interesting case is where we come across a global system which is very different from ours, and where, according to our translation manual, the two systems have the same empirical content and are irreconcilable. In such a case, it would be tempting to question the assumption that shared terms mean the same in the two systems. The reason is that a theoretical term gets at least some of its meaning holistically from its use in the theory to which it belongs. In other words, we may assume that shared terms are ambiguous in the way suggested by Quine. If there are any syntactic incompatibilities, they should not be taken seriously. Perhaps it would even be 'absurd' to stick to a translation manual which makes the other theory incompatible, but empirically equivalent, with ours. Besides, the socalled Principle of Charity (WO 59fn) might be taken to count against such a manual.

Nevertheless, such a translation manual might exist, and it is not obvious that it must violate the requirement of fluent and effective dialogue. I suggest that it is still an open question whether there could be interesting cases of alternative global systems which have the same empirical content and are logically incompatible. However, cases involving incompatibilities like that between GC and $G \neg C$ are not interesting enough.

\section{Scepticism.}

Quine says that cases of incompatible systems can always be 'reduced' to cases of compatible systems by the method mentioned above (PT 97). Maybe so. But if the systems in question are incompatible, they must already be formulated in one and the same language. ${ }^{14}$ The 'reduction' consists in an extension of the vocabulary of the language and the creation of a new theory formulated in the extended language. However, the reduced theory is not reduced away. The two original systems are still incompatible.

If the two original systems are also empirically equivalent in the sense that "whatever observation would be counted for or against the one theory counts equally for or against the other', it seems that everything which counts for one of the systems counts equally against the very same system-since it also counts for an incompatible system. In this way, underdetermination seems to lead to scepticism.

\footnotetext{
${ }^{14}$ This is pointed out by Davidson. He writes: 'Quine's two theories can belong to, and be stated in, the same language; indeed, they must be if we are to understand the claim that the theories conflict', 'The structure and content of truth', The Journal of Philosophy, vol. 87 (1990), 279-328, p. 306.
} 
Quine attempts to avoid scepticism by insisting that truth is immanent to our system of the world. Given the situation in which some system of the world is empirically equivalent but incompatible with ours, Quine asks: 'Can we say that one [of the systems], perhaps, is true, and the other therefore false, but that it is impossible in principle to know which?' His answer is that this would be a mistake; rather 'it would be our place to insist on the truth of our laws and the falsity of the other theory where it conflicts'. The reason is that, on his naturalistic view, 'there is no extra-theoretic truth, no higher truth than the truth we are claiming or aspiring to as we continue to tinker with our system of our truth from within' (EES 327).

But it seems to me that there are really two different questions here, and that Quine does not keep them clearly apart. One question is whether we should call our system (or the other system) true. The other is whether we can know that our system is true. Quine answers the first question. Our system is the system we believe is true. As long as we believe that it is true, we should call it true. But if we also believe that all our evidence for this system is equally good evidence against it, perhaps we should conclude that we do not know that our system is true. If underdetermination is unavoidable, we might also conclude that it is impossible to know which system is true.

However, this sceptical conclusion presupposes that the incompatible systems in question are empirically equivalent in Quine's general sense. As I have argued above, this presupposition is unwarranted.

Besides, even if our system of the world is incompatible with some empirically equivalent system, the two systems may have a lot in common. If so, there is no need to adopt a sceptical attitude to the common part. ${ }^{15}$ For example, if the systems are our old friends $G C$ and $G \neg C$, the incompatibility argument should not make us sceptical about $G$. If both systems are equally coherent, we may have good reasons to accept $G$, but no good reasons either to accept or reject $C$. To the extent that there are more interesting cases of incompatible and empirically equivalent systems, we may also be entitled to more interesting degrees of scepticism. But as I have argued above, the existence of such cases is dubious.

\section{Ecumenism and sectarianism.}

On the other hand, it seems entirely plausible to assume that logically compatible but quite different global systems might have the same empirical content (relative to us now) as our

\footnotetext{
${ }^{15}$ Hence, the scepticism I have discussed here should be described as partial. In fact, there is some reason to think that Quine would accept a partial scepticism of this kind. In his reply to Gibson, Quine writes: 'What can be known of the world is the common denominator of all the world systems, logically reconciled, that conform to all possible observation' (RG 156). It seems to follow that, if there is no common denominator, nothing can be known of the world. In the case of empirically equivalent systems, there must be a common denominator. This includes, at the very least, the common empirical content of the different systems. Perhaps the systems may overlap in other ways too; the underdetermination thesis does not say anything in particular about the extent of such overlap.
} 
own global system of the world. If so, one might find it reasonable to regard all such systems as equally true. In Quine's words:

If we subscribe to one of them as true, we can call them all true and view them as different descriptions of one and the same world. We are no strangers, after all, to strange languages. If this be relativism, make the most of it (RA 295).

This is an expression of Quine's ecumenic stance (RG 156). In other passages, as we have seen above, he adopts instead a sectarian position, according to which we should regard our own system as true and the other systems as false or meaningless. For example:

we should indeed recognize [all the systems] as equally well warranted. We might even oscillate between them, for the sake of a richer perspective on nature. But we should still limit the ascription of truth to whichever theory formulation we are entertaining at the time, for there is no wider frame of reference (TT 29).

Quine is both an empiricist and a naturalist. He claims that his empiricism motivates the ecumenic position, whereas naturalism motivates sectarianism (RG 156, PT 99). In one place, he also says that the question of which position to take is 'a question of words' (PT 101). What are we to make of this?

As far as I can see, it is not a question of words. Rather, it is a fundamental question of ontology. It has to do with what to count as real. Such questions have to be dealt with from the point of view of what we believe, i.e. from the point of view of our global system of the world. This means that Quine should accept the sectarian position.

The ecumenic position can only be acceptable for someone who accepts an empiricist theory of truth. According to an empiricist theory of truth, roughly speaking, a sentence is true if it is entailed by an empirically adequate theory. But Quine does not accept an empiricist theory of truth. ${ }^{16}$ The empiricism he accepts is a theory of evidence and of meaning (see e.g. OR 75).

We may also argue against ecumenism as follows. Suppose that two systems $A$ and $B$ are irreconcilable and have the same empirical content. Suppose also that we believe, ecumenically, that they are both true. Surely, we should then believe that the conjunction $A B$ is true. Presumably, these three theories - the conjunction and its two conjuncts - are empirically equivalent in the sense that they have the same empirical content. But they are not empirically equivalent in the sense that "whatever observation would be counted for or against the one theory counts equally for or against the other'. The conjunction $A B$ is less simple than its conjuncts, and it may also be less coherent. Quine says that $A B$ should not

\footnotetext{
${ }^{16}$ Compare TT 39, RB and also Quine's comments to my paper 'Quine, empiricism, and truth', in Alex Orenstein and Petr Kotátko (eds.) Knowledge, Language and Logic - Questions for Quine, forthcoming.
} 
be accepted under such conditions. He rejects what he calls 'the tandem solution' (PT 99). Now, it seems to me that if we should not accept the conjunction, neither should we accept both conjuncts. This undermines the ecumenical position. Strangely enough, Quine seems to think that it is all right for us to accept both $A$ and $B$ ('we account both theories separately true'), and at the same time not accept the tandem theory $A B$ (PT 99-101). In my opinion, this is not an attractive position. I find it unintelligible.

\title{
Relativism.
}

So, from a Quinean point of view, sectarianism is right-as Quine himself seems to think most of the time. But sectarianism seems to be a form of relativism. It is a form of relativism in that makes truth in some way relative to theory. Quine sometimes expresses this by saying that truth is immanent. For example, he says that

\footnotetext{
it is a confusion to suppose that we can stand aloof and recognize all the alternative ontologies as true in their several ways, all the envisaged worlds as real. It is a confusion of truth with evidential support. Truth is immanent, and there is no higher. We must speak from within a theory, albeit any of various (TT 21-2).
}

In this passage, Quine rejects ecumenism and accepts sectarianism. Much the same thought seems to be expressed in the following remark:

\footnotetext{
It is $[\ldots]$ when we turn back into the midst of an actually present theory [...] that we can and do speak sensibly of this or that sentence as true. Where it makes sense to apply 'true' is to a sentence couched in the terms of a given theory, and seen from within the theory (WO 24).
}

This very passage is cited by Davidson as something that seems to point in the direction of relativism. ${ }^{17}$ However, in the same context, Davidson writes:

\begin{abstract}
I had worried that when he [Quine] wrote that truth is 'immanent' he was expressing the idea that truth is relative not only to a language, but also in some further way. He assures me that no other relativization is implied beyond the familiar, and unavoidable, relativization to a language (ibid.).
\end{abstract}

But Davidson also points to "the fact that "theory" and "language" are not to be clearly distinguished in Quine's writings' (ibid.), so there may still be some kind of relativization of truth to theory. However, in his response to Davidson, Quine seems to agree that relativization to language is all that is involved, and he refers to 'our common foe who

\footnotetext{
17 'What is Quine's view of truth?', Inquiry, 37, No. 4 (1994), 437-40, p. 437.
} 
would relativize truth to theory'. The role of theory 'was not in legislating truth, but in clarifying the theoretical sentence' (RD 498).

This is not easy to understand. For example, what is meant by the statement that truth is 'relative to language'? One might suppose that a given sentence can belong to more than one language and that it can have different meanings in different languages; hence, it may be true in one language and false in another. Maybe this is what Davidson has in mind. But Quine seems to hold that a given theoretical sentence (or utterance) belongs to just one language or one theory. Thus, in his response to Davidson, he quotes his own statement that 'a sentence is meaningless except relative to its own theory' (RD 498, WO 24). This indicates that each (theoretical) sentence has a theory it can call its own. ${ }^{18}$ If so, there is no point in saying that a sentence can have different meanings in different theories or in different languages. A sentence is intelligible, and hence disquotable, only as seen from within its own theory or system of the world. 'Disquotation explains truth only insofar as the disquoted sentence is intelligible', as Quine puts it (RD 498).

So truth is not relative in the sense that one and the same sentence can be true relative to one theory and false relative to another. In this sense, at least, Quine is not a relativist. However, on Quine's view, truth still seems to be relative to theory in the sense that the truth of a sentence presupposes a whole theory or system of the world to which the sentence belongs and from which it gets all the meaning it has. From our point of view, a sentence of another culture, in which a different system of the world is accepted, is meaningless and therefore neither true nor false. Of course, it can be given a meaning in the sense that we may devise a successful translation manual from the alien language into ours, but according to Quine's thesis of the indeterminacy of translation, many different manuals may be equally successful and none of them is objectively the correct one. We may say that the alien sentence is true relative to a given translation manual, but what is relative here is meaning rather than truth. Truth is still somehow related to our own theory. Disquotation can only be applied within one's own language. This is also why ecumenism has to be rejected.

Quine's idea that truth is immanent can hardly be taken as a purely epistemological claim. The point is not merely that in order to find out whether a given sentence is true, we have to take much of our own system of the world for granted; we have to relate the sentence to other sentences that we accept as true. This is unavoidable, but I take it that Quine's claim is also, and primarily, ontological. Of course, the claim is not that a sentence is true if and only if it is implied by our system of the world. Parts of our system may be false. Rather, I believe that the claim can be roughly stated as follows: a necessary

\footnotetext{
${ }^{18}$ Presumably, a theoretical sentence in one theory (language) may have the same syntactic form as a sentence in another, but, if so, they are still to be reckoned as two distinct sentences. Notice, also, that there is still a sense in which 'truth is relative to language', namely that an explicit definition of truth, in non-semantic terms, can only be constructed for a particular language (in accordance with the method invented by Tarski).
} 
condition for a sentence of our language to be true is that most of the sentences in our system of the world are true. And similarly for other cultures. In this sense, I suggest, truth is relative to theory. ${ }^{19}$

It might be objected that Quine's naturalism is violated if we allow the addition: 'similarly for other cultures'. By saying this, we may seem to transcend our own system of the world in an illegitimate way. But I think this should be acceptable to Quine. What we say about other cultures is still said from within our own system of the world. We are not violating Quine's thesis that 'it is a confusion to suppose that we can stand aloof and recognize all the alternative ontologies as true in their several ways, all the envisaged worlds as real' (TT 21). We are not saying that all the systems are true. We are not in this sense adopting a transcendent position outside all systems including our own.

What we do is, I believe, similar to what Quine himself does when he says that the sectarian 'is as free as the ecumenist to oscillate between the two theories [two empirically equivalent systems of the world] for the sake of added perspective from which to triangulate on problems' (PT 100). Suppose the sectarian says, from within our system of the world, that a certain sentence $S$ is true. According to Quine, the sectarian also recognizes that he may 'oscillate' to some alien system, which is just as warranted as ours, but from within which he would no longer regard $S$ as true. I think we may legitimately express this by saying that truth is relative to theory. (Personally, however, I am not so sure that it would really be possible to 'oscillate' in this way.)

As far as I can see, then, the relativism I attribute to Quine is consistent with his naturalism. However, Quine has explicitly rejected relativism on the ground that it is paradoxical (EES 327).

Truth, says the cultural relativist, is culture-bound. But if it were, then he, within his own culture, ought to see his own culture-bound truth as absolute. He cannot proclaim cultural relativism without rising above it, and he cannot rise above it without giving it up (EES 327-8).

But it is not clear to me that this is really paradoxical. To say that truth is 'culture-bound' is, in this context, much the same as to say that it is 'immanent', and Quine does not seem to find this paradoxical. The relativist says that truth is relative to a culture or a system of the world ('culture-bound'). Does he need to see this very thesis itself as 'absolute'? Yes and no. He cannot make a statement and relativize it at the same time. This would be much the

\footnotetext{
${ }^{19}$ In his recent paper 'Quine's philosophy: A brief sketch', in L.E. Hahn and P.A. Schilpp (eds.), The Philosophy of W.V. Quine, expanded edition (Chicago and LaSalle, Ill., Open Court, 1998), Roger Gibson says that 'he [Quine] believes truth is an immanent notion, relativized to a theory (or a language)'. In his reply to Gibson (RGQ 685), Quine accepts relativization to language but not to theory; he says 'I grant language but balk at theory'. However, his reason seems to be that '[a] theory that I hold may turn out false' (ibid.). This is uncontroversial but, I think, irrelevant. I suspect that Quine would not allow that most of the sentences in our system of the world may turn out to be false. The truth-or-falsity of a theory or sentence in our system of the world may still presuppose the truth of most of the system.
} 
same as making the statement and taking it back in the same breath. Nothing would be accomplished. However, he may make second order statements about his own statements. Such a second order statement may be the following: 'While relativism is absolutely true, someone from another culture with a very different system of the world may say that the relativism I proclaim is meaningless or merely true-relative-to my system, just as I say that his utterances can be true-relative-to his system even though they are in fact meaningless or at most true relative to some translation manual I might devise'.

A slightly different view, which can also be described as a form of 'cultural relativism', is expressed in the following passage:

\footnotetext{
Might another culture, another species, take a radically different line of scientific development, guided by norms that differ sharply from ours but that are justified by their scientific findings as ours are by ours? And might these people predict as successfully and thrive as well as we? Yes, I think that we must admit this as a possibility in principle; that we must admit it even from the point of view of our own science, which is the only point of view I can offer. I should be surprised to see this possibility realized, but I cannot picture a disproof (TT 181).
}

Surely, this has a relativistic ring. We may note that this passage describes a different kind of underdetermination from those identified above. In this case, we are asked to imagine two systems of the world which are equally successful in a certain way. As far as I can see, this does not imply that they have the same empirical content, nor that they are empirically equivalent in Quine's more general sense. Again, however, it seems that we can 'proclaim cultural relativism without rising above it' ${ }^{20}$

\section{List of abbreviations of Quine's works.}

CB 'Comment on Bergström', in R. Barrett and R. Gibson (eds.) Perspectives on Quine (Cambridge, Mass. and Oxford, Blackwell, 1990), 53-4.

CNS 'Comments on Newton-Smith', Analysis, 39, No. 2 (1979), 66-7.

EES 'On empirically equivalent systems of the world', Erkenntnis, 9 (1975), 313-28.

OR Ontological Relativity and Other Essays (New York: Columbia University Press, 1969).

PT Pursuit of Truth (Cambridge, Mass., Harvard University Press, 1990).

RA 'Relativism and absolutism', The Monist, 67 (1984), 293-96.

RB 'Response to Bergström', Inquiry, 37, No. 4 (1994), 496-8.

RD 'Response to Davidson', Inquiry, 37, No. 4 (1994), 498-500.

\footnotetext{
${ }^{20}$ I wish to thank Björn Eriksson, Roger Gibson, Folke Tersman, and Peter Pagin for valuable comments on the first draft of this paper.
} 
RG 'Reply to Roger F. Gibson, Jr.', in L. Hahn and P. Schilpp (eds.), The Philosophy of W.V. Quine (La Salle, Ill., Open Court, 1986), 155-57.

RGQ 'Reply to Roger F. Gibson, Jr.', in L. Hahn and P. Schilpp (eds.), The Philosophy of W.V. Quine, expanded edition, (Chicago and La Salle, Ill., Open Court, 1998), 667-83.

RIT 'On the reasons for indeterminacy of translation', The Journal of Philosophy, 67 (1970) 178-83.

SS From Stimulus to Science (Cambridge, Mass., Harvard University Press, 1995).

TT Theories and Things (Cambridge, Mass., Belknap Press, 1981), second printing.

WB The Web of Belief (with J.S. Ullian) 2d ed. (New York, Random House, 1978).

WO Word and Object (Cambridge, Mass., M.I.T. Press, 1960). 